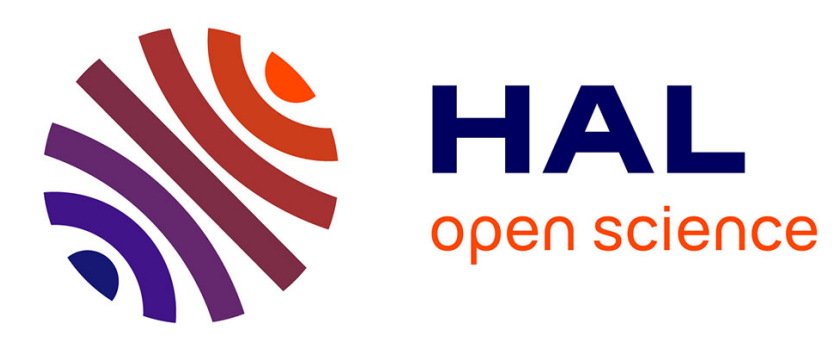

\title{
An Inelastic Thermal Spike Model to calculate Ion Induced Desorption Yields
}

M. Bender, H. Kollmus, H. Reich-Sprenger, M. Toulemonde, W. Assmann

\section{To cite this version:}

M. Bender, H. Kollmus, H. Reich-Sprenger, M. Toulemonde, W. Assmann. An Inelastic Thermal Spike Model to calculate Ion Induced Desorption Yields. The Seventh International Symposium on Swift Heavy Ions in Matter, Jun 2008, Lyon, France. hal-00256301

\section{HAL Id: hal-00256301 https://hal.science/hal-00256301}

Submitted on 25 Aug 2008

HAL is a multi-disciplinary open access archive for the deposit and dissemination of scientific research documents, whether they are published or not. The documents may come from teaching and research institutions in France or abroad, or from public or private research centers.
L'archive ouverte pluridisciplinaire HAL, est destinée au dépôt et à la diffusion de documents scientifiques de niveau recherche, publiés ou non, émanant des établissements d'enseignement et de recherche français ou étrangers, des laboratoires publics ou privés. 


\title{
An Inelastic Thermal Spike Model to calculate Ion Induced Desorption Yields
}

\author{
M. Bender ${ }^{\mathrm{a}, *}$, H. Kollmus ${ }^{\mathrm{b}}$, H. Reich-Sprenger ${ }^{\mathrm{b}}$, \\ M. Toulemonde ${ }^{\mathrm{c}}, \mathrm{W}$. Assmann ${ }^{\mathrm{a}}$ \\ ${ }^{a}$ Ludwig-Maximilians-Universität München, Garching, Germany \\ ${ }^{\mathrm{b}}$ Gesellschaft für Schwerionenforschung, Darmstadt, Germany \\ ${ }^{\mathrm{c}}$ CIMAP, Caen, France
}

\begin{abstract}
Ion induced desorption is a severe luminosity limitation for low charge state heavy ion accelerators. Therefore, it was intensively investigated in dedicated experiments during recent years. Several experimental results were obtained providing numerous desorption yields for different ion beam parameters and different materials as well as surface treatments. The heavy ion induced desorption was identified as a pure surface cleaning effect. Nevertheless it was shown that the yields have a strong link to the irradiated material. The initial desorption yield decreases during the irradiation reaching a dynamic equilibrium. Desorption yields of several hundred molecules per incident ion from one monolayer adsorbed gas can not be explained with the geometrical cross section of the projectile. Therefore we have expanded the inelastic Thermal Spike Model to describe the process as thermal desorption from a microscopic heated region. The obtained results of this extended model represent very well the numbers from many experimental studies.
\end{abstract}

Key words: ion-induced desorption, ion-solid interaction, inelastic Thermal Spike 


\section{Introduction}

The performance of heavy ion accelerators is strongly dependent on the vacuum base pressure. Especially in the case of high current low charge state acceleration vacuum break-downs have been observed $(1 ; 2 ; 3)$. With the low charge state one takes advantage of a high space charge limit but with the disadvantage of a comparatively high ionization cross section. Low charge state beam ions can get ionized in collisions with the residual gas. These beam ions get lost in or after the next dipole magnet and collide with the chamber wall. Due to the collision gas is desorbed from the chamber wall leading to a pressure increase in the accelerator vacuum system. This results in higher charge exchange rates and thus to even more desorption and finally to the complete breakdown of the acceleration cycle.

For the GSI future project FAIR (4), the existing heavy ion synchrotron SIS18 as the injector is expected to deliver $10^{12} \mathrm{U}^{28+}$ Ions per second in a 4 Hertz acceleration mode. Therefore an experimental program was started at GSI to measure ion-induced desorption yields of different accelerator relevant materials (5) and to find a suited low desorbing material for the so called beam loss collimators (6). These experiments helped to understand the physical processes of the ion induced desorption and the main results are summarized in the following:

\footnotetext{
* Corresponding author

Email address: m.bender@gsi.de (M. Bender).
} 
- In all experiments a cleaning of the target during irradiation was observed. After a fluence between $5 \cdot 10^{12}$ and $2 \cdot 10^{13}$ ions $/ \mathrm{cm}^{2}$ the desorption reached a minimum value dominated by $\mathrm{H}_{2}$ desorption (5). The amount of the desorbed gas was always of the order of a monolayer of gas within the beam spot area $\left(\approx 10^{13}\right.$ gas molecules per $\left.\mathrm{mm}^{2}\right)$. The conditioning effect is frequently called beam scrubbing. Due to the required fluence, beam scrubbing is no solution for accelerator conditioning at GSI.

- The desorbed gas was dominated by carbon monoxide (CO), Hydrogen $\left(\mathrm{H}_{2}\right)$ and carbon dioxide $\left(\mathrm{CO}_{2}\right)$. These gas species typically dominate any good UHV environment. Cleaning is very effective for $\mathrm{CO}$ and $\mathrm{CO}_{2}$ and less effective for $\mathrm{H}_{2}$.

- The desorption yield depends on the projectile energy. For the same projectile on the same sample the yield scales with the electronic energy loss of the projectile in the sample to the power of 2-3.

- Isolating materials show quite larger desorption yields than conducting materials for the same projectile energy loss (7). For example the desorption yield of copper may be increased at least by a factor of five by an oxide layer on the surface.

- Materials analysis has shown that the target composition does not measurably change during irradiation and beam scrubbing.

- Also highly pure materials show sometimes high desorption yields (e.g., rhodium has $\eta \approx 1000$ whereas gold has $\eta \approx 100$ for the same irradiation conditions). This support the assumption that the desorbed gas is predominately coming from the surface of the sample and sputtering of an oxide layer or impurities play a minor role.

- During in-situ bake-out of the samples it was shown that at $350^{\circ} \mathrm{C}$ a huge amount of CO leaves the target. After such a bake-out the sample showed 
a desorption yield as low as a beam-cleaned sample after some $10^{13}$ ions / $\mathrm{cm}^{2}$.

All these experimental results showed that heavy ion induced desorption is predominately a surface effect but has a strong link to the underlaying material. Earlier assumptions, that diffused and sputtered impurities from the bulk lead to the high pressure rises, could be disproved. Only $\mathrm{H}_{2}$ diffuses from the bulk to the surface. Therefore it is less effectively removed leading to a final desorption yield level which cannot be further lowered. Typically the ratio between initial and final desorption yields is in the range of 15-20.

Based on the experimental results, we describe the observed high desorption yields as a pure surface effect due to a thermal activated process, which is also supported by the fact that a larger electron-phonon coupling leads to a larger desorption yield. In our model calculation the ion induced desorption results from a microscopic thermal desorption of the heated spot around the ion impact. The temperature rise in the spot is calculated using the inelastic Thermal Spike Model (8) and is basically given by the energy loss of the incident ion to the sample and the target properties.

\section{Model Calculation}

The calculation of the ion induced desorption is based on the idea that the dynamic equilibrium of adsorption and desorption on a surface is interfered by the heating of the microscopic area around the ion impact. Since the inelastic Thermal Spike Model predicts well the results of various track formation (9) and sputter yield measurements (10), it was extended to describe desorption 
of gas molecules from surfaces within the above mentioned picture. Due to the ion impact the temperature around the ion track can increase by orders of magnitudes during 10 to 100 picoseconds leading to an enhanced thermal gas desorption. Within the Thermal Spike code the evolution of the temperature of the electronic and the atomic subsystem can be calculated in space and time $(\mathrm{T}(\mathrm{r}, \mathrm{t}))(8 ; 11)$. In the references phenomena like sputtering, track formation and phase transitions were correlated with the temperature resulting from the Thermal Spike code. Here, the temperature map of the samples surface was combined with the equations for thermal desorption to achieve absolute desorption yields.

The Thermal Spike calculations are performed one-dimensionally. However the resulting desorption yield values from the surface are obtained by integrating over the circular area $\left(A=\int 2 \pi r d r\right)$. The circular symmetry is obvious for perpendicular ion impact as performed in the experiments. The desorption yield per incident ion is given from the temperature of the atomic lattice by

$$
\eta=\int_{0}^{t_{\max }} \int_{0}^{r_{\max }} \nu_{0}(T(r, t)) \cdot \tilde{n}(r, t) \cdot \exp \left(-\frac{E_{\text {des }}}{k_{B} \cdot T(r, t)}\right) d r d t
$$

where $\nu_{0}(T(r, t))=\frac{k \cdot T(r, t)}{h}$ ( $h$ is the Planck constant) is the oscillation frequency of the adsorbate of approximately $10^{13} \mathrm{~s}^{-1}, \tilde{n}(r, t)$ is the time dependant surface coverage of the area, $E_{\text {des }}$ the binding energy of the adsorbate and $k_{B}$ the Boltzmann constant. The limits of the integrals depend on the active radius of the ion, where the temperature is sufficiently high to trigger desorption. This radius is typically in the range of a few $10 \mathrm{~nm}$. As mentioned above, the time duration is of the order of some 10 to 100 picoseconds. Both are strongly depending on material properties like, e.g., thermal conductivity and specific heat. 
Based on experimental results the surface is considered to be covered by one monolayer of gas. For first calculations we concentrate on chemically inert surfaces and assume a typical binding energy of $0.4 \mathrm{eV}$ for a gas molecule bound to the metallic surface.

\section{Results}

In the following, as an example, calculations for $1.4 \mathrm{MeV} / \mathrm{u} \mathrm{Xe}$ on $\mathrm{Cu}$ will be shown. The projectile charge states in the experiments $(20+/ 21+)$ are by a factor of one third smaller than the equilibrium charge state $(29+)$. For the sake of simplicity, the calculations are performed using the electronic energy loss of $1.4 \mathrm{MeV} / \mathrm{u} \mathrm{Xe} \mathrm{e}^{29+}$ in $\mathrm{Cu}$, which is $3792 \mathrm{eV} / \AA$ as given by SRIM2006 (12). Results of the calculations will be compared to measured yields with 1.4 $\mathrm{MeV} / \mathrm{u} \mathrm{Xe}^{20+/ 21+}$, and variations of input parameters will be discussed. As a boundary condition the initial temperature of the sample is $293 \mathrm{~K}$ and a surface coverage of one monolayer gas is assumed. Fig. 1 shows the temperature evolution of the electronic subsystem in space (x axis) and time (y axis) after the ion impact. The temperature of the surface is given by the color. It can be seen that in the order of $10^{-15}$ seconds a maximum temperature of around $2.5 \cdot 10^{5} \mathrm{~K}$ is reached. Even at distances of some $10 \mathrm{~nm}$ the electronic subsystem reaches temperatures of around $\approx 1000 \mathrm{~K}$. After $10^{-15}$ seconds the temperature starts to decrease. Meanwhile the energy couples to the atomic lattice as displayed in Fig. 2. Here, a temperature of $900 \mathrm{~K}$ is easily reached within one $\mathrm{nm}$ radius. But even at a distance of $25 \mathrm{~nm}$ from the ion impact typical bake-out temperatures (13) of $500 \mathrm{~K}$ are exceeded. Inserting this temperature map in the Boltzmann equation (Eq. (1)), the distribution of desorption over 
time and distance from the impact point can be calculated as shown in Fig. 3. The color code gives the number of desorbed particles per time step as a function of the radius. The background desorption at room temperature has been subtracted in this plot. Therefore the integration over radius and time gives the desorption yield, i. e. the total number of particles per incident ion.

\section{Comparison of Different Materials}

In the following, different targets will be compared for a certain set of boundary conditions: the electron density and the electron-phonon coupling are fixed to 1.5 valence electrons as proposed by Dufour et al. (14), which seems to be a good compromise for a non-crystalline but conducting target. The surface coverage is set to be one monolayer and the binding energy of the gas molecules is $0.4 \mathrm{eV}$ for all targets. A comparison of temperatures for the electronic subsystems of the different targets is displayed in Fig. 4. From the different distributions of the electronic temperature, which is directly linked to the electronic energy loss, the amount of desorbed gas cannot be extracted. Nevertheless, it can be seen that for $\mathrm{Au}$ same temperatures are reached at larger distances than for Rh. In all cases the electronic system is starting to cool down after roughly $10^{-13}$ seconds due to electron-phonon coupling. The temperature distribution of the atomic system for the different samples is shown in Fig. 5. Here one can clearly see that, close to the ion track, Rh (bottom) is the hottest for the longest time duration. Au (middle) reaches the lowermost maximum temperatures. However, the medium heated region up to $40 \mathrm{~nm}$ where temperatures higher than $400 \mathrm{~K}$ are reached for a comparatively long time still contributes to the yield. The amount of released gas as a function of radius 
and time is shown in Fig. 6. For this parameter set desorption yields of 185 for copper, 165 for gold and 3400 for rhodium are calculated.

\section{Discussion of the Input Parameters}

This model is by far no ab-initio calculation. In the following the input parameters will be discussed to justify a certain set of parameters for the calculation.

\subsection{Surface Coverage}

The surface coverage with adsorbed gas molecules enters linearly into the calculation: double surface coverage doubles the yield. On the other hand a minimized coverage leads to lowest desorption yields as shown experimentally (5). Since the measured surface coverage after a regular UHV bake-out was always in the order of one monolayer this value is also used for the calculations.

\subsection{Binding Energy}

The binding energy is very critical for the model since it enters exponentially into the calculation. Fig. 7 illustrates the influence of the binding energy on the desorption yield for different temperatures. Here, the correction factor for the desorption yield is plotted versus the deviation from the calculated yield using a binding energy of $0.4 \mathrm{eV}$. Close to room temperature the dependence on the binding energy is much more pronounced as compared to higher temperatures. In reality the binding energy strongly depends on the binding system, namely on the sample materials and surface properties as well as on the adsorbed 
gas species. These binding energies are only partially known from TDS spectroscopy. Here, a value of $0.4 \mathrm{eV}$ seems to be fairly reasonable but one has to consider that this is an uncertainty of the model, especially since the same binding energy is used for all sample materials.

\subsection{Electron-Phonon Coupling}

The number of electrons participating in the electron-phonon coupling depends on the sample properties. Reasonable values used to simulate track formation and sputter yield measurements are one to two participating electrons (15). Fig. 8 shows the variation in the electronic and lattice temperature evolution as well as in the desorption yield for $\mathrm{Cu}$ using 1, 1.5 and 2 electrons. It is seen that for an increasing number of electrons the electronic subsystem is faster cooling down, since the energy is stronger coupled to the lattice. As a consequence the atomic system gets hotter leading to a higher desorption yield. For Xe ion incidence on $\mathrm{Cu}$, as an example, the desorption yield is 120 for 1 electron, 185 for 1.5 and 350 for 2 electrons. Gold is less sensitive to the variation of the electron number, here the yields vary from 135 to 211. For Rh the yields vary between 1900 and 4000. With a mean value of 1.5 electrons, which is also stated by track formation and sputter yield measurements, the experimentally found desorption yields are best represented.

\subsection{Energy Loss}

The incident ion is not in its equilibrium charge state. Therefore the real energy loss is less than the one used in the calculation. An estimation by the 
CASP code $(16 ; 17)$ gives roughly a 1.36 times lower energy loss for Xe in $\mathrm{Cu}$ and a 1.25 times lower value for $\mathrm{Xe}$ in $\mathrm{Au}$. On the other hand, charge state equilibrium is reached within $10 \mathrm{~nm}$ (18) and an energy loss varying along the ion track has to be considered which is not possible in this one-dimensional model. The absolute yield, but not the yield relation between the different materials will be changed if more exact energy loss values are used.

\section{Comparison with Experimental Results}

With the following parameters the calculated values may now be compared to the experimental results. Here, the initial temperature is $\mathrm{T}_{0}=293 \mathrm{~K}$, the surface is covered by 1 monolayer, the binding energy is $0.4 \mathrm{eV}$ and 1.5 electrons contribute to the electron-phonon coupling.

In table 1 , the calculated values for $\mathrm{Au}, \mathrm{Rh}$ and $\mathrm{Cu}_{2} \mathrm{O}$ overestimate the measured yields at least by a factor of two. The higher experimental value of copper may be explained by the sample surface, which was found to be very rough and contaminated by hydrogen from the lapping and etching procedure, even though there was no oxide at all. In contrast, the gold and the rhodium sample were proven to have smooth and clean surfaces. The overestimation might be partly explained by the charge state dependent energy loss. For example, a 1.36 times lower energy loss for $\mathrm{Xe}$ in $\mathrm{Cu}$ would decrease the calculated yield from 185 to 95 (Fig. 9).

The lower three lines compare the calculation with values of older experiments (1). Here the experimental values are higher than the calculated ones by a factor of two. It has to be mentioned that in these experiments the copper sample surfaces were oxidized since they where stored in normal atmosphere 
for several months.

\section{$7 \quad$ Energy Loss Scaling}

In many track formation and sputter yield measurements as well as in desorption yield measurements a quadratic dependence on the electronic energy loss was found (19). This indicates a thermally moderated process. Therefore, desorption yields for xenon on copper and gold were calculated for different energy losses corresponding to different impact energies. Fig. 9 shows the result of the calculation for copper. The points represent the calculated desorption yield values for different projectile energies and, hence, energy losses. The line is a quadratic fit to the data. Thus, also the model calculation reveals a quadratic scaling of the desorption yield with energy loss.

\section{Copper Oxide Calculation}

The inelastic Thermal Spike Model works best for metals. For insulators the mean free path of the electrons is the critical parameter, which may be related to the band gap energy. For the experimentally investigated copper oxide $\mathrm{Cu}_{2} \mathrm{O}$, the band gap energy is $2 \mathrm{eV}$ and, therefore, the mean free path length is $7 \cdot 10^{-9} \mathrm{~m}$. With this input the desorption yield is calculated to be roughly 10000. One has, however, to consider that the values of the thermal conductivity as well as the electro-phonon mean free path are not well known for $\mathrm{Cu}_{2} \mathrm{O}$. 


\section{Conclusion \& Outlook}

The extension of the inelastic Thermal Spike Model to thermal desorption gives for the first time calculated values for heavy ion induced desorption. Even though the model parameters have some uncertainty, the ranking of the desorption yields of the different materials is confirmed as well as the quadratic scaling with the energy loss. In consequence, ion induced desorption in the investigated energy range can be described mainly as a thermally activated process, triggered by the microscopic heating of a single ion impact. As a next step, the influence of the input parameter should be studied in more detail and the real gas composition as well as the different binding energies of the different gas species on different surfaces should be considered.

\section{Acknowledgements}

We acknowledge the support of the European Community-Research Infrastructure Action under the FP6 "Structuring the European Research Area" program (DIRACsecondary-Beams, contract number 515873).

\section{References}

[1] H. Kollmus, M. Bender, M. C. Bellachioma, E. Mahner, A. Krämer, L. Westerberg, E. Hedlund, O. B. Malyshev and H. Reich-Sprenger, AIP CP 773 (2005) 207

[2] E. Mahner, I. Efthymiopoulos, J. Hansen, E. Page, and H. Vincke, Phys. Rev. ST Accel. Beams 7103202 (2004) 
[3] S. Y. Zhang, Internal Note C-A / AP / 67, BNL, New York (1999)

[4] D. Krämer, Proceedings of RuPAC, Novosibirsk Russia (2006) 1

[5] M. Bender, PhD Thesis, University of Frankfurt (2008)

[6] C. Omet, P. Spiller, J. Stadlmann, Proceedings of EPAC, Edinburgh Scotland (2006) 211

[7] M. Bender, H. Kollmus, W. Assmann, Nucl. Instr. and Meth. B 256 (2007) 387

[8] C. Dufour, A. Audouard, F. Beuneu, J. Dural, J. P. Girard, A. Hairie, M. Levalois, E. Paumier and M. Toulemonde, J. Phys. Condensed Matter 5 (1993) 4573

[9] M. Toulemonde, Ch. Dufour, Z. Wang, E. Paumier, Nucl. Instr. and Meth. $112(1996) 26$

[10] M. Toulemonde, W. Assmann, C. Trautmann, and F. Grüner, Phys. Rev. Lett. 88 (2002) 057602

[11] M. Toulemonde, C. Dufour, and E. Paumier, Phys. Rev. B 46 (1992) 14362

[12] J. F. Ziegler: Monte-Carlo-Code SRIM, Ver. 06, New York (2006)

[13] J. B. Hudson in: J. M. Lafferty (Ed.), Foundations of Vacuum Science and Technology, Wiley-Interscience, (1998) 619

[14] Ch. Dufour, Z. G. Wang, M. Levalois, P. Marie, E. Paumier, F. Pawlak, M. Toulemonde, Nucl. Instr. and Meth. 107 (1996) 218

[15] H. D. Mieskes, W. Assmann, F. Grüner, H. Kucal, Z. G. Wang, and M. Toulemonde, Phys. Rev. B 67 (2003) 155414

[16] P. L. Grande, G. Schiwietz, Phys. Rev. A 58 (1998) 3796

[17] G. dze M. Azevedo, P.L. Grande, G. Schiwietz, Nucl. Instr. and Meth. B $164(2000) 203$

[18] F. Grüner, F. Bell, W. Assmann, and M. Schubert, Phys. Rev. Lett. 93 
(2004) 213201

[19] A. W. Molvig et al., Phys. Rev. Lett. 98 (2007) 064801 


\section{Table 1}

Comparison of experimental and calculated desorption yields (molecules / ion). The displayed charge state was used in the experiments while the calculations were done for charge state equilibrium.

\begin{tabular}{|l|l|l|}
\hline collision system & experiment & calculation \\
\hline $1.4 \mathrm{MeV} / \mathrm{u} \mathrm{Xe}^{21+}: \mathrm{Cu}$ & $290 \ldots 360$ & 185 \\
$1.4 \mathrm{MeV} / \mathrm{u} \mathrm{Xe}{ }^{21+}: \mathrm{Au}$ & 90 & 165 \\
$1.4 \mathrm{MeV} / \mathrm{u} \mathrm{Xe}^{20+}: \mathrm{Rh}$ & $915 \ldots 1286$ & 3400 \\
$1.4 \mathrm{MeV} / \mathrm{u} \mathrm{Xe}^{21+}: \mathrm{Cu} 2 \mathrm{O}$ & 1530 & 10000 \\
$1.4 \mathrm{MeV} / \mathrm{u} \mathrm{C}^{2+}: \mathrm{Cu}$ & 10 & 5 \\
$1.4 \mathrm{MeV} / \mathrm{u} \mathrm{Cr}{ }^{7+}: \mathrm{Cu}$ & 150 & \\
$1.4 \mathrm{MeV} / \mathrm{u} \mathrm{Pb}{ }^{27+}: \mathrm{Cu}$ & 800 & 525 \\
$4.2 \mathrm{MeV} / \mathrm{u} \mathrm{Pb}^{53+}: \mathrm{Au}$ & 800 & \\
\hline
\end{tabular}




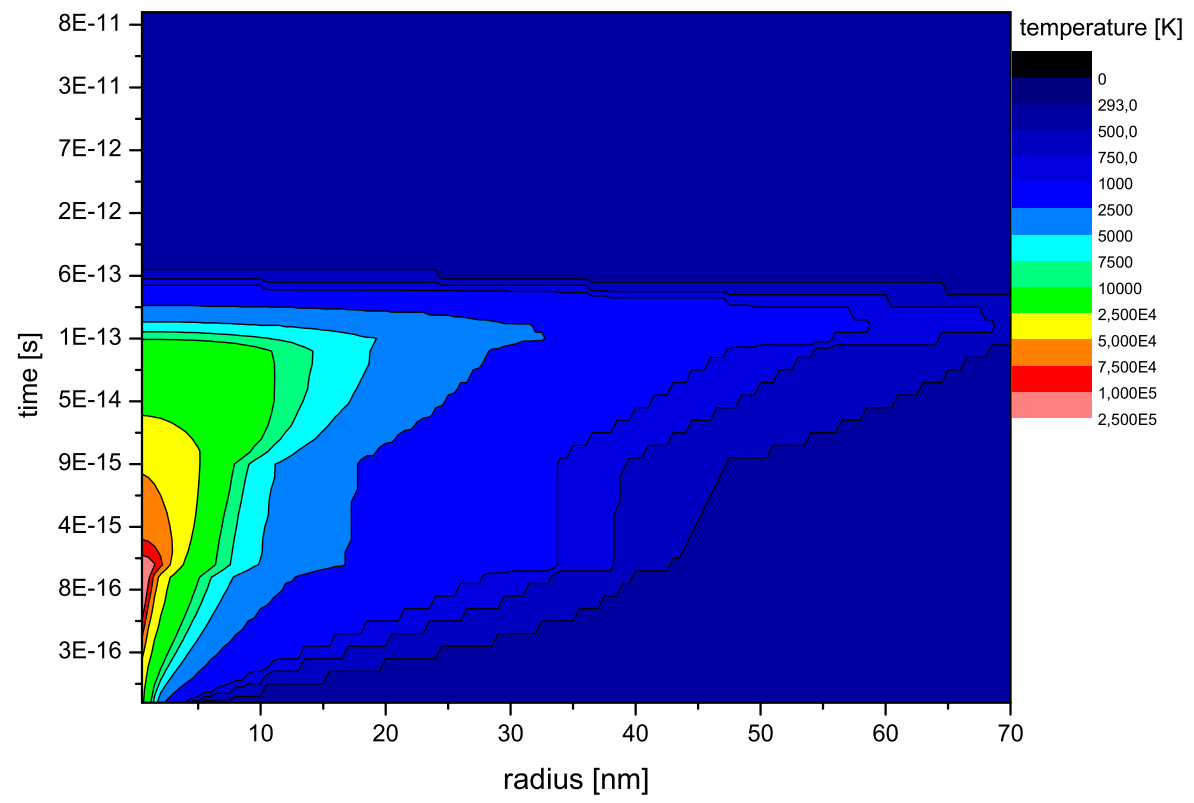

Fig. 1. Temperature evolution of the electronic subsystem in time after and distance from the ion impact of a Xe ion with $1.4 \mathrm{MeV} / \mathrm{u}$ in $\mathrm{Cu}$. 


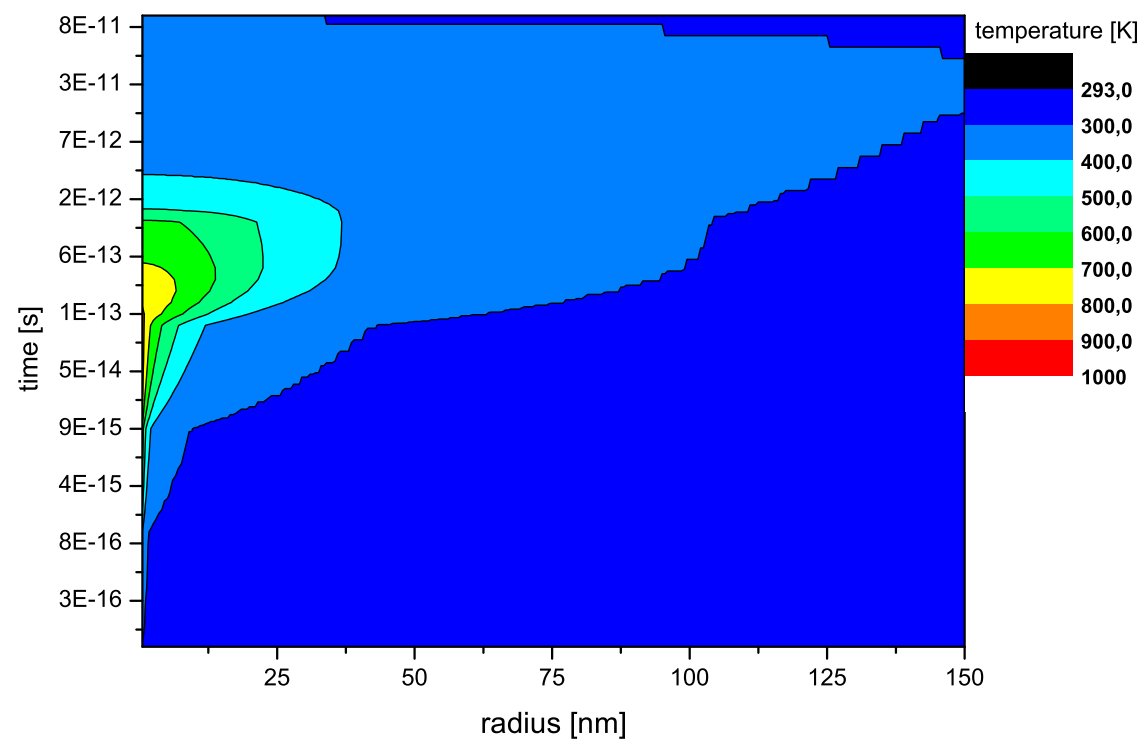

Fig. 2. Temperature of the atomic subsystem of $\mathrm{Cu}$ after $1.4 \mathrm{MeV} / \mathrm{u}$ Xe ion impact (corresponding to Fig. 1). 


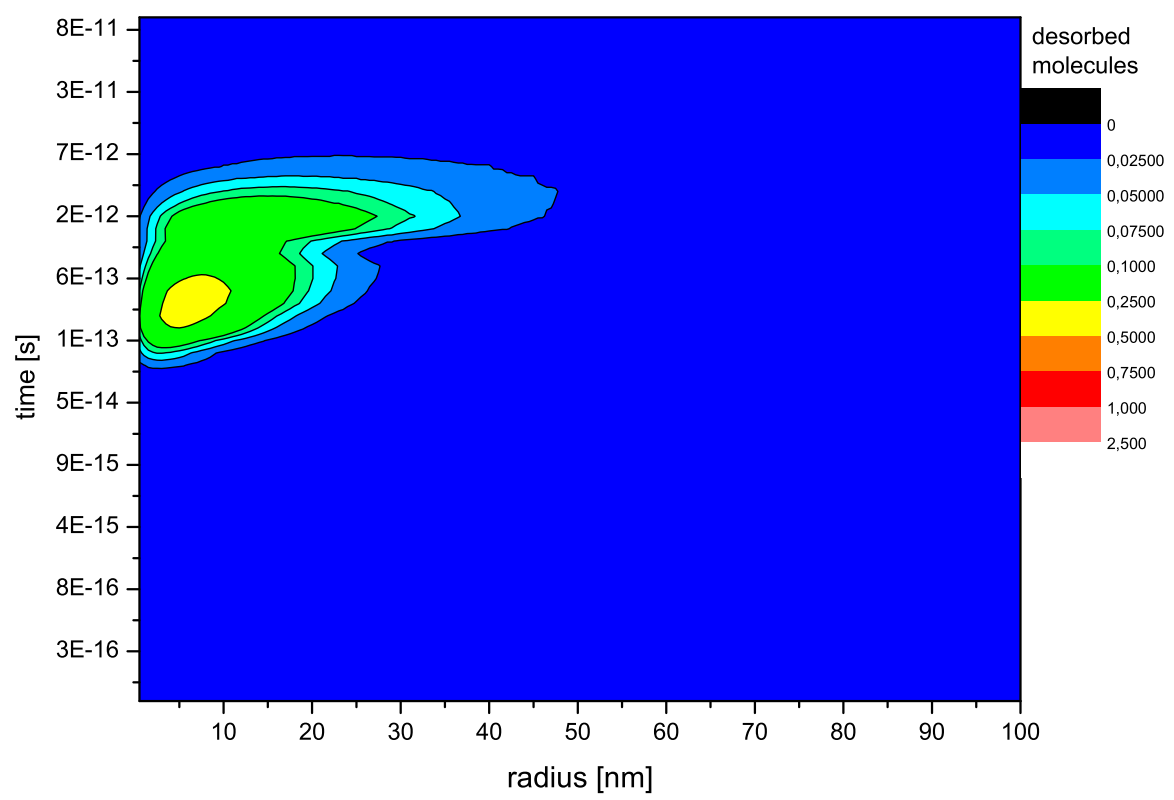

Fig. 3. Desorbed particles as a function of radius and time after the collision (corresponding to Fig. 1 and 2). The radial distribution is integrated over $2 \pi$ to calculate absolute yields. 


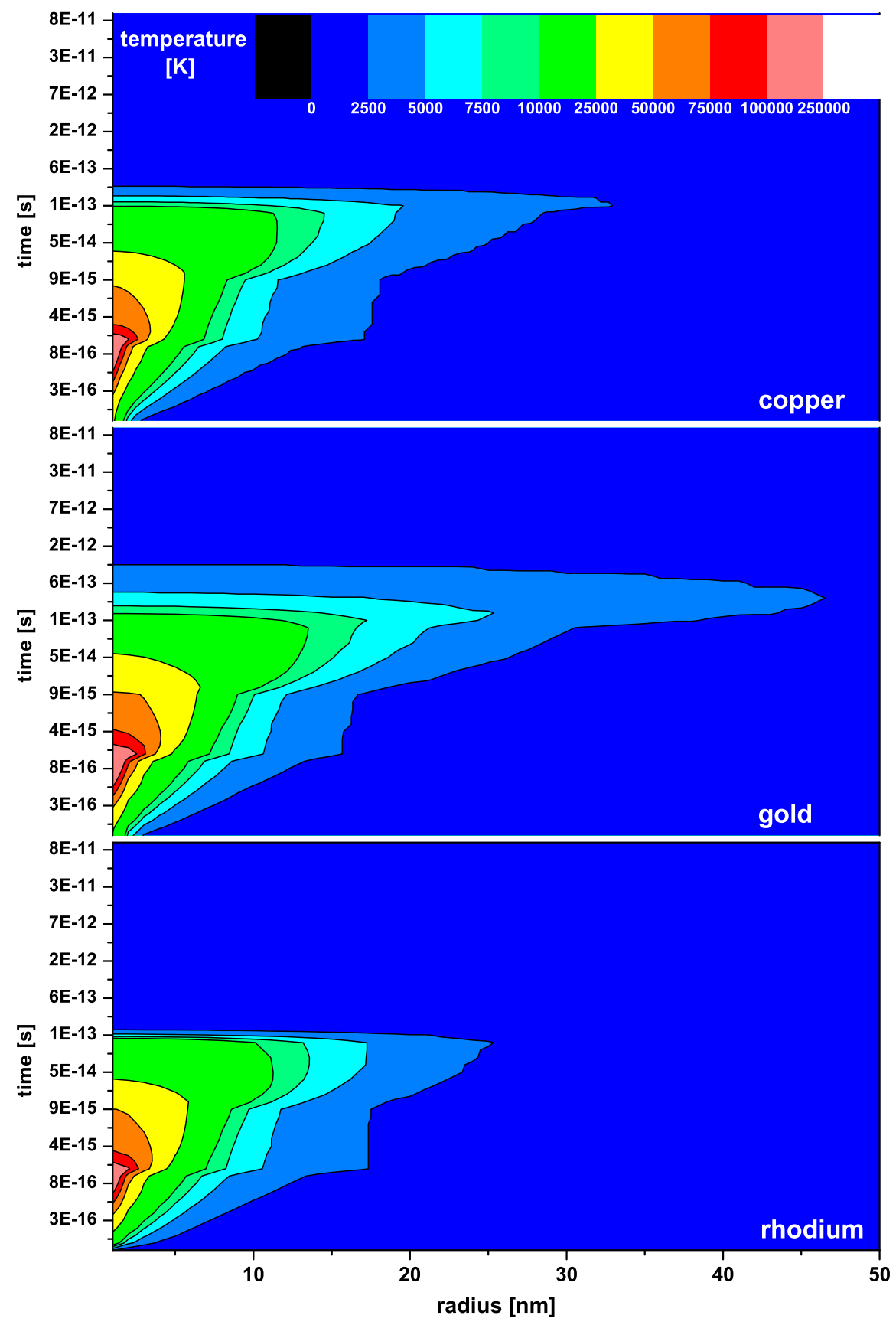

Fig. 4. Comparison of the temperature of the electronic subsystem for $\mathrm{Cu}, \mathrm{Au}$ and $\mathrm{Rh}$ after $1.4 \mathrm{MeV} / \mathrm{u}$ Xe impact. 


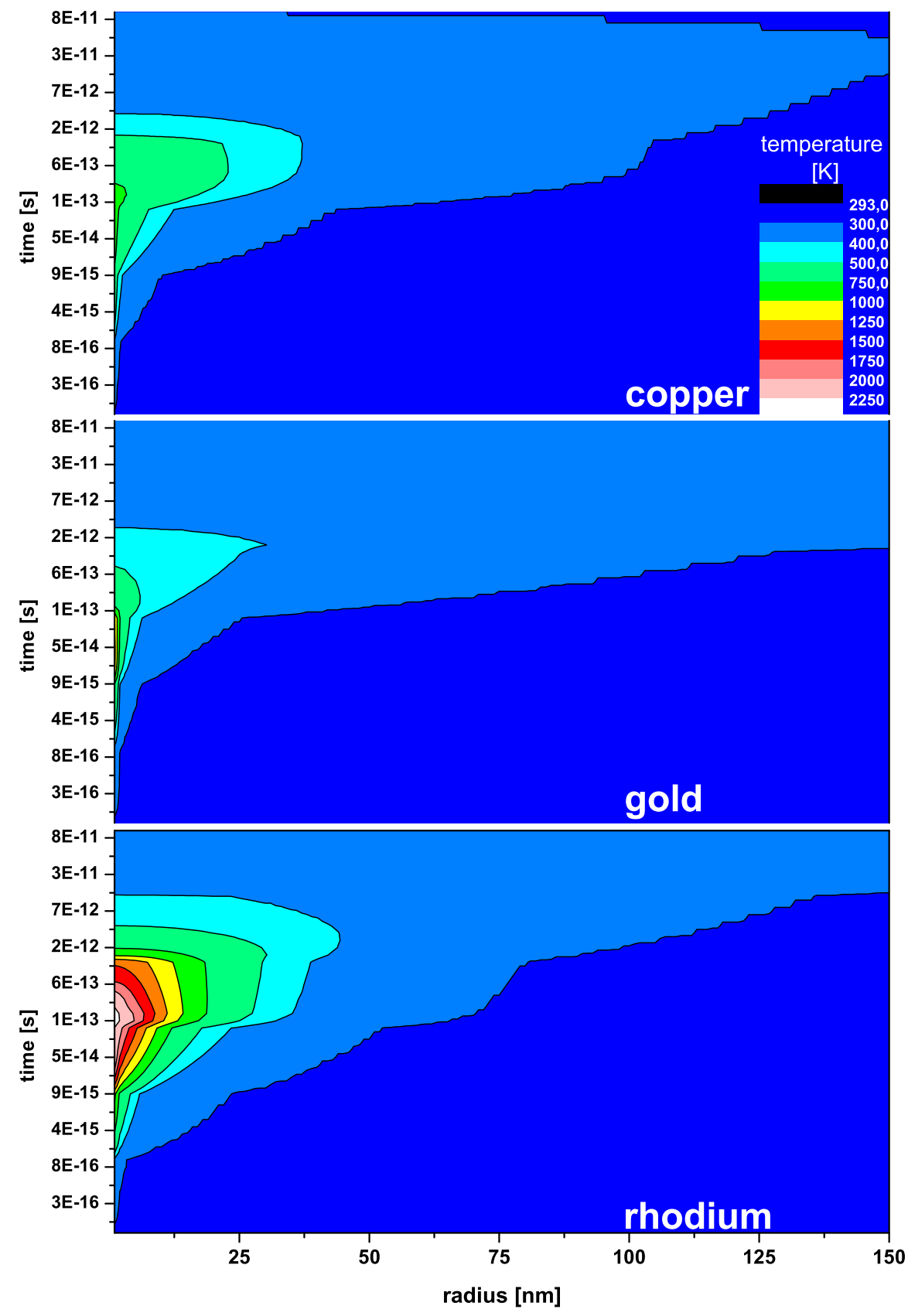

Fig. 5. Temperature of the atomic subsystem corresponding to Fig. 4. 


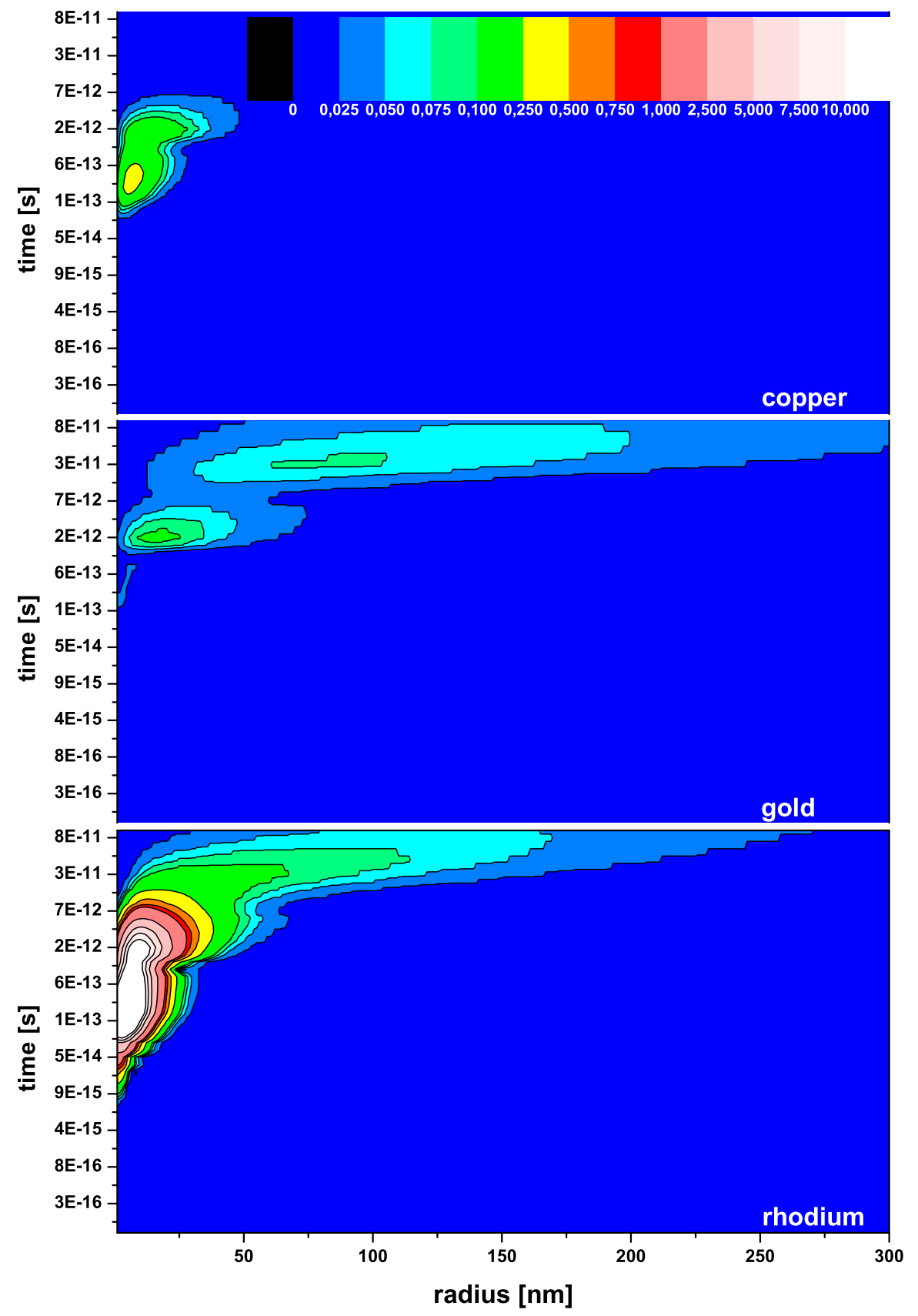

Fig. 6. Desorbed molecules as a function of time and radius after the ion impact, corresponding to Fig. 5. The radial distribution is integrated over $2 \pi$ to calculate absolute yields. 


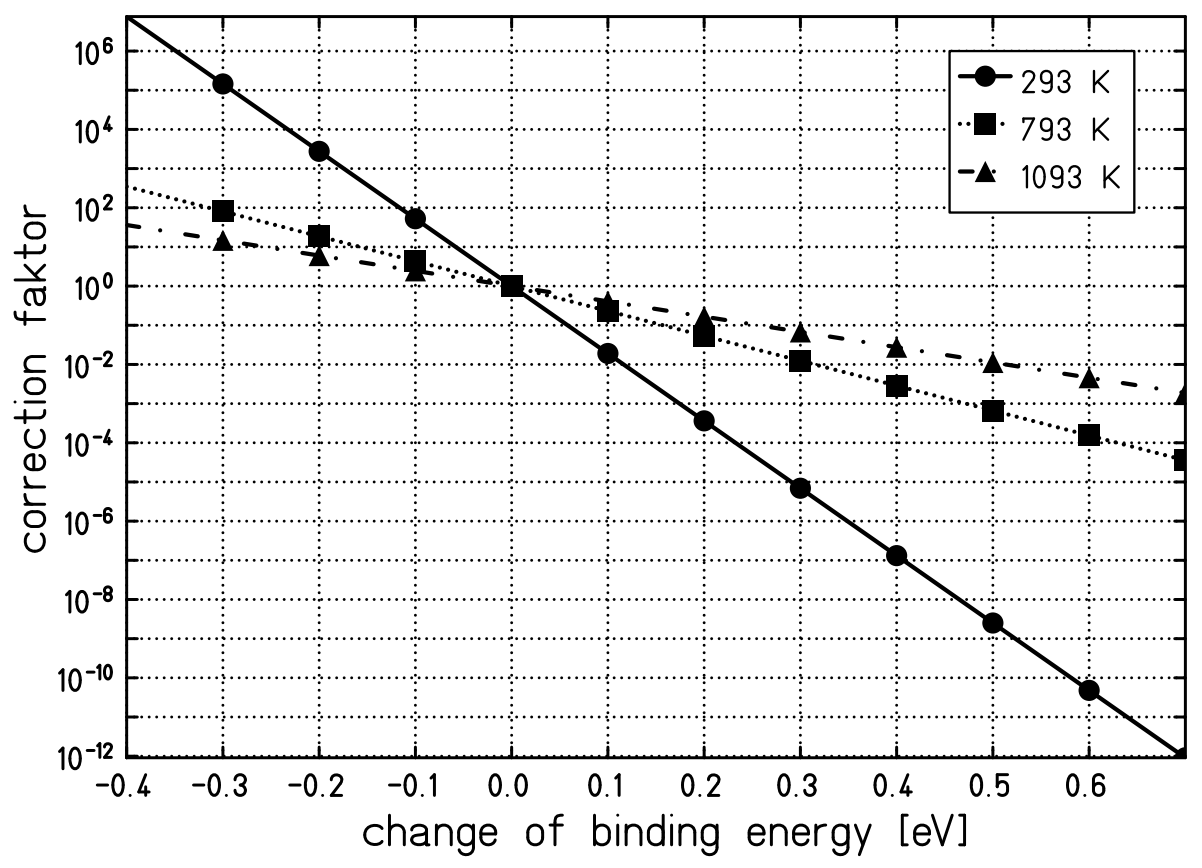

Fig. 7. Yield correction factor for different binding energies and temperatures. 


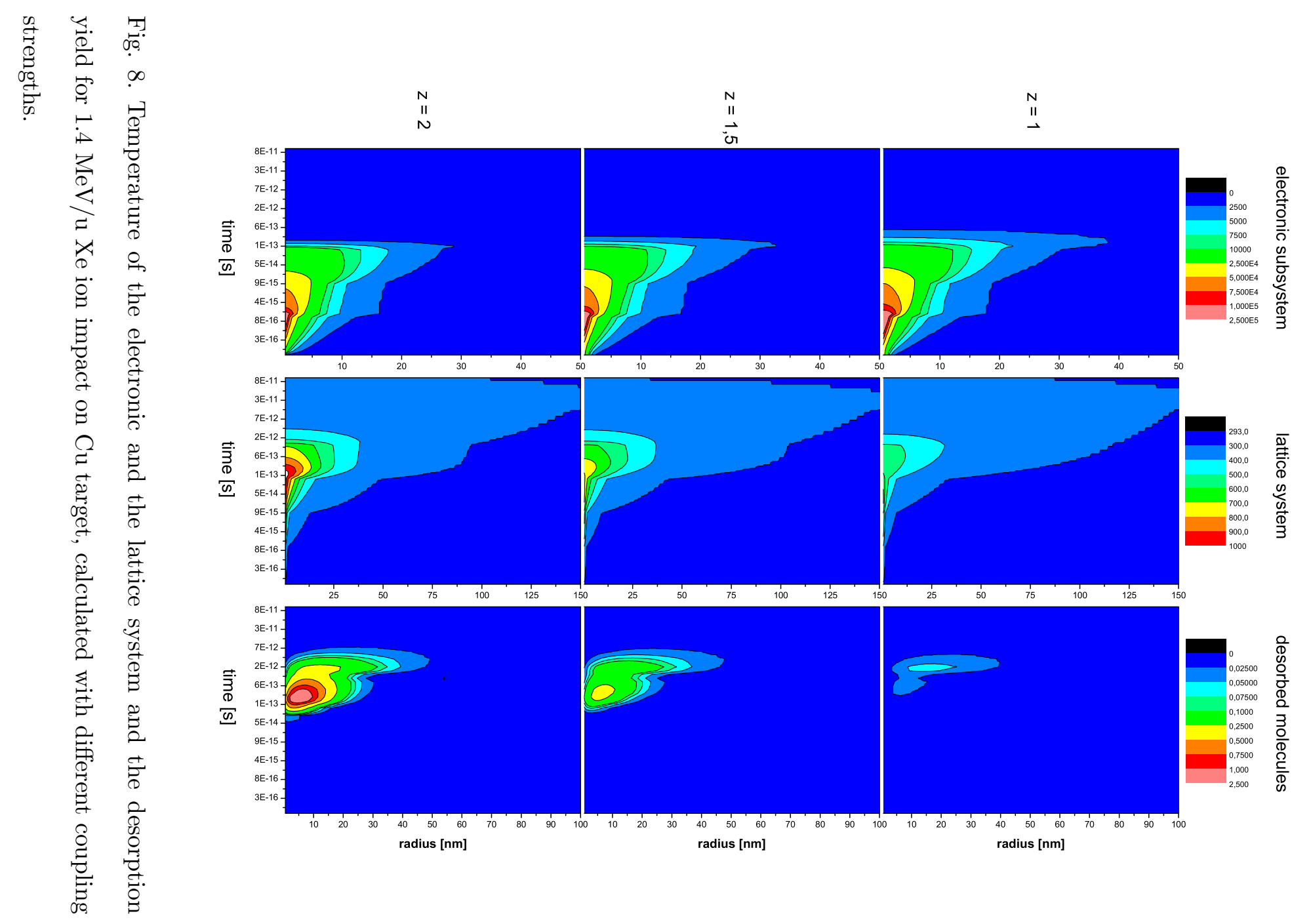




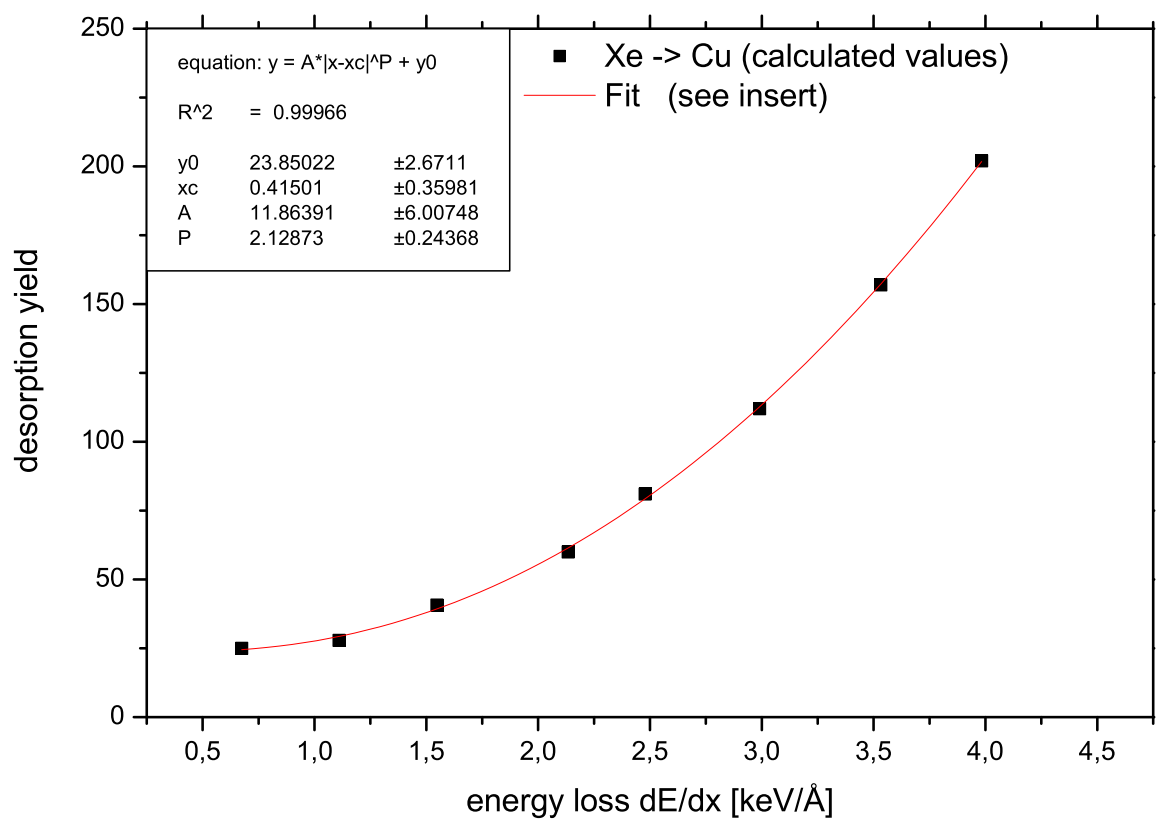

Fig. 9. Calculated desorption yield for different incident energies (energy loss) of Xe ions on $\mathrm{Cu}$. 